Bangladesh J. Sci. Ind. Res. 41(3-4), 147-158, 2006

\title{
Decay of Temperature Fluctuations in Homogeneous Turbulence before the Final Period for the Case of Multi-Point and Multi-Time in a Rotating System
}

\author{
M. S. Alam Sarker and M. A. K. Azad \\ Department of Mathematics, University of Rajshahi, Rajshahi-6205, Bangladesh
}

\begin{abstract}
Using Deissler's approach the decay of temperature fluctuations in homogeneous turbulence before the final period for the case of multi-point and multi-time in a rotating system in presence of dust particle is studied and have considered correlation between fluctuating quantities at two and three point. Two and three point correlation equations in a rotating system is obtained and the set of equations is made to determinate by neglecting the quadruple correlations in comparison to the second and third order correlations. The correlations are converted to spectral form by taking their Fourier transforms. Finally integrating the energy spectrum over all wave numbers, the energy decay law of temperature fluctuations in homogeneous turbulent before the final period for the case of multi-point and multi-time in a rotating system is obtained.
\end{abstract}

\section{Introduction}

Deissler $^{1,2}$ developed a theory for homogeneous turbulence which was valid for times before the final period. Following Deissler's theory Loeffler and Deissler ${ }^{3}$ studied the decay of temperature fluctuations in homogeneous turbulence before the final period. In their study, they presented the theory which is valid during the period for which the quadruple correlation terms are neglected compared to the 2nd and 3rd order correlation terms. Using Deissler's same theory Kumar and Patel ${ }^{4}$ studied the first-order reactions in homogeneous turbulence before the final period for the case of multi-point and single-time. The problem $^{4}$ is extended to the case of multipoint and multi-time concentration correlation by Kumar and Patel $^{5}$ and also the numerical result of ${ }^{5}$ carried out by Patel. ${ }^{6}$ Following
Deissler's approach Sarker and Islam studied the decay of MHD turbulence before the final period for the case of multi-point and multi-time. $^{7}$ M. A. Islam and M. S. A. Sarker $^{8}$ also studied the first-order reactant in MHD turbulence before the final period of decay for the case of multi-point and multitime. Sarker and L. Rahman ${ }^{9}$ studied the decay of temperature fluctuations in MHD turbulence before the final period. Sarker and Islam $^{10}$ also studied the decay of temperature fluctuations in homogeneous turbulence before the final period for the case of multi-point and multi-time. Azad and Sarker ${ }^{11}$ studied the decay of MHD turbulence before the final period for the case of multi-point and multi-time in presence of dust particle. 
M. A. K. Azad and S. A. Sarker ${ }^{12}$ also studied the decay of dusty fluid MHD turbulence before the final period in a rotating system for the case of multi-point and multi-time. S. Sultana and S. A. Sarker ${ }^{13}$ discussed the Decay of homogeneous dusty fluid turbulence before the final period. Further work along this same line has more recently been done by M. A. K. Azad and M. S. Alam Sarker. ${ }^{14,15}$

In their approach, they considered two and three point correlations and neglecting fourth- and higher-order correlation terms compared to the second-and third-order correlation terms.

In this paper the Deisslers ${ }^{1,2}$ method is used and we have studied the decay of temperature fluctuations in homogeneous turbulence before the final period for the case of multi-point and multi-time in a rotating system.

\section{Correlation and spectral equations}

For an incompressible fluid with constant properties and for negligible frictional heating, the energy equation may be written as

$$
\frac{\partial \widetilde{T}}{\partial t}+\widetilde{u}_{i} \frac{\partial \widetilde{T}}{\partial x_{i}}=\frac{k}{\rho C_{p}} \frac{\partial^{2} \tilde{T}}{\partial x_{i} \partial x_{i}}
$$

where $\widetilde{T}$ and $\widetilde{u}_{i}$ are instantaneous values of temperature and velocity; $\mathrm{k}$, thermal conductivity; $\rho$, fluid density; $C_{p}$ heat capacity at constant pressure; $\mathrm{x}_{\mathrm{i}}$, space coordinate; $\mathrm{t}$, time and the repeated subscripts are summed from 1 to 3 .
Breaking these instantaneous values into time average and fluctuating components as

$$
\widetilde{T}=\langle T\rangle+T \text { and } \widetilde{u}_{i}=\left\langle u_{i}\right\rangle+u_{i}
$$

where $\mathrm{T}$, temperature fluctuation from time average ; $\langle T\rangle$, time average value of temperature; $u_{i}$, velocity fluctuation from time average; $\left\langle u_{i}\right\rangle$, time average value of velocity.

From the condition of homogeneity it follows that $\frac{\partial\langle T\rangle}{\partial x_{i}}=0$, and in addition the usual assumption is made that $\langle T\rangle$ is independent of time and that $\left\langle u_{i}\right\rangle=0$. Thus equation (1) becomes

$$
\frac{\partial T}{\partial t}+u_{i} \frac{\partial T}{\partial x_{i}}=\left(\frac{v}{p_{r}}\right) \frac{\partial^{2} T}{\partial x_{i} \cdot \partial x_{i}}
$$

where $p_{r}=v / \gamma$, Prandtl number; $v$, kinematic viscosity and $\gamma=\frac{k}{\rho C_{p}}$.

Equation (2) is assumed to hold at the arbitrary point $p$. For the point $p^{\prime}$ the corresponding equation can be written

$$
\frac{\partial T^{\prime}}{\partial t^{\prime}}+u_{i}^{\prime} \frac{\partial T^{\prime}}{\partial x_{i}^{\prime}}=\left(\frac{v}{p_{r}}\right) \frac{\partial^{2} T^{\prime}}{\partial x_{i}^{\prime} \partial x_{i}^{\prime}}
$$

The points $\mathrm{p}$ and $\mathrm{p}^{\prime}$ are separated by the vector $\hat{r}$ is shown below

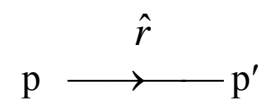


Multiplying equation (2) by $T^{\prime}$, equation (3) by $\mathrm{T}$ and taking ensemble average, result in

$$
\begin{aligned}
& \frac{\partial\left\langle T T^{\prime}\right\rangle}{\partial t}+\frac{\partial\left\langle T T^{\prime} u_{i}\right\rangle}{\partial x_{i}}=\left(\frac{v}{p_{r}}\right) \frac{\partial^{2}\left\langle T T^{\prime}\right\rangle}{\partial x_{i} \partial x_{i}} \\
& \frac{\partial\left\langle T T^{\prime}\right\rangle}{\partial t^{\prime}}+\frac{\partial\left\langle T T^{\prime} u_{i}^{\prime}\right\rangle}{\partial x_{i}^{\prime}}=\left(\frac{v}{p_{r}}\right) \frac{\partial^{2}\left\langle T T^{\prime}\right\rangle}{\partial x_{i}^{\prime} \partial x_{i}^{\prime}}
\end{aligned}
$$

with the continuity equation,

$$
\frac{\partial u_{i}}{\partial x}=\frac{\partial u_{i}^{\prime}}{\partial x_{i}^{\prime}}=0
$$

The angular bracket has been used to denote an ensemble average.

Using the transformations

$$
\begin{aligned}
\frac{\partial}{\partial x_{i}} & =-\frac{\partial}{\partial r_{i}}, \frac{\partial}{\partial x_{i}^{\prime}}=\frac{\partial}{\partial r_{i}},\left(\frac{\partial}{\partial t}\right)_{t^{\prime}} \\
& =\left(\frac{\partial}{\partial t}\right)_{\Delta t}-\frac{\partial}{\partial \Delta t}, \frac{\partial}{\partial t^{\prime}}=\frac{\partial}{\partial \Delta t}
\end{aligned}
$$

into equation (4) and (5), one obtains

$$
\begin{aligned}
& \frac{\partial\left\langle T T^{\prime}\right\rangle}{\partial t}-\frac{\partial\left\langle u_{i} T T^{\prime}\right\rangle}{\partial r_{i}}(-r,-\Delta t, t+\Delta t)+ \\
& \frac{\partial\left\langle T T^{\prime} u_{i}^{\prime}\right\rangle}{\partial r_{i}}(\hat{r}, \Delta t, t)=2\left(\frac{v}{p_{r}}\right) \frac{\partial^{2}\left\langle T T^{\prime}\right\rangle}{\partial r_{i} \partial r_{i}}-\cdots \\
& \frac{\partial\left\langle T T^{\prime}\right\rangle}{\partial \Delta t}+\frac{\partial\left\langle u_{i} T T^{\prime}\right\rangle}{\partial r_{i}}(-\hat{r},-\Delta t, t+\Delta t) \\
& =\left(\frac{v}{p_{r}}\right) \frac{\partial^{2}\left\langle T T^{\prime}\right\rangle}{\partial r_{i} \partial r_{i}}
\end{aligned}
$$

It is convenient to write this equation in spectral form by use of the following threedimensional Fourier transforms as follows. ${ }^{16}$

$$
\left\langle T T^{\prime}(\hat{r}, \Delta t, t)\right\rangle=\int_{-\infty}^{\infty}\left\langle\tau \tau^{\prime}(\hat{K}, \Delta t, t)\right\rangle \exp [\hat{i}(\hat{K} \cdot \hat{r})] d \hat{K}
$$

$$
\left\langle u_{i} T T^{\prime}(\hat{r}, \Delta t, t)\right\rangle=\int_{-\infty}^{\infty}\left\langle\phi_{i} \tau \tau^{\prime}(\hat{K}, \Delta t, t)\right\rangle \exp [\hat{i}(\hat{K} \cdot \hat{r})] d \hat{K}
$$

It is more obvious by interchanging the point $\mathrm{p}$ and $\mathrm{p}^{\prime}$ that

$$
\begin{aligned}
& \left\langle u_{i}^{\prime} T T^{\prime}(\hat{r}, \Delta t, t)\right\rangle=\left\langle u_{i} T T^{\prime}(-\hat{r},-\Delta t, t+\Delta t)\right\rangle \\
& =\int_{-\infty}^{\infty}\left\langle\phi_{i} \tau \tau^{\prime}(-\hat{K},-\Delta t, t+\Delta t)\right\rangle \exp [\hat{i}(\hat{K} . \hat{r})] d \hat{K}
\end{aligned}
$$

where $\hat{K}$ is known as a wave number vector and magnitude of $\hat{K}$ has the dimension 1/length and can be considered to be the reciprocal of an eddy size.

Substitution of equations (9)-(11) into equations (7) and (8) leads to the spectral equations.

$$
\begin{aligned}
& \frac{\partial\langle\tau \tau\rangle}{\partial t}+2\left(\frac{v}{p_{r}}\right) k^{2}\left\langle\tau \tau^{\prime}\right\rangle=i K_{i}\left[\left\langle\phi_{i} \tau \tau^{\prime}(\hat{K}, \Delta t, t)\right\rangle\right. \\
& \left.-\left\langle\phi_{i} \tau \tau^{\prime}(-\hat{K},-\Delta t, t+\Delta t)\right\rangle\right] \\
& \frac{\partial\left\langle\tau \tau^{\prime}\right\rangle}{\partial \Delta t}+2\left(\frac{v}{p_{r}}\right) k^{2}= \\
& -i K_{i}\left\langle\phi_{i} \tau \tau^{\prime}\right\rangle(-\hat{K},-\Delta t, t+\Delta t)
\end{aligned}
$$


In equations (12)-(13) the quantity $\tau \tau^{\prime}(\hat{K})$ may be interpreted as a temperature fluctuation "energy" contribution of thermal eddies of size $1 / \mathrm{k}$. The time derivative of this "energy' as a function of the convective transfer to the wave numbers and the "dissipation" due to the action of the thermal conductivity. The term on the right hand side of equation (12) is also called transfer term while the $2^{\text {nd }}$ term on the left hand side is the "dissipation" term.

\section{Three-point, Three-time correlation and} spectral equations

Considering three points $\mathrm{p}, \mathrm{p}^{\prime}, \mathrm{p}^{\prime \prime}$ separated by the vectors $\hat{r}$ and $\hat{r}^{\prime}$, we write the Naviour Stocke's equation for turbulent flow of incompressible fluid in a rotating system at the point $p$, energy equations at the points $\mathrm{p}^{\prime}$ and $\mathrm{p}^{\prime \prime}$.

$$
\begin{aligned}
& \sum_{\hat{r}^{\prime}}^{\hat{r}^{\prime} \rightarrow \mathrm{p}^{\prime}} \\
& \frac{\mathrm{p}_{j}^{\prime \prime}}{\partial t}+\frac{\partial}{\partial x_{i}}\left(u_{j} u_{i}\right)=-\frac{1}{\rho} \frac{\partial p}{\partial x_{j}}+v \frac{\partial^{2} u_{j}}{\partial x_{i} \partial x_{i}} \\
& -2 \in_{m i j} \Omega_{m} u_{j} \\
& \frac{\partial T^{\prime}}{\partial t}+u_{i}^{\prime} \frac{\partial T^{\prime}}{\partial x_{i}^{\prime}}=\left(\frac{v}{P_{r}}\right) \frac{\partial^{2} T^{\prime}}{\partial x_{i}^{\prime} \partial x_{i}^{\prime}} \\
& \text { and } \frac{\partial T^{\prime \prime}}{\partial t}+u_{i}^{\prime \prime} \frac{\partial T^{\prime \prime}}{\partial x_{i}^{\prime \prime}}=\left(\frac{v}{P_{r}}\right) \frac{\partial^{2} T^{\prime \prime}}{\partial x_{i}^{\prime \prime} \partial x_{i}^{\prime \prime}}
\end{aligned}
$$

Multiplying equations (14) - (16) by $T^{\prime} T^{\prime \prime}$, $u_{j} T^{\prime \prime}$ and $u_{j} T^{\prime}$ respectively and then taking ensemble average, we obtained

$$
\begin{aligned}
& \frac{\partial\left\langle u_{j} T^{\prime} T^{\prime \prime}\right\rangle}{\partial t}+\frac{\partial\left\langle u_{j} T^{\prime} T^{\prime \prime} u_{i}\right\rangle}{\partial x_{i}}=-\frac{1}{\rho} \frac{\partial\left\langle P T^{\prime} T^{\prime \prime}\right\rangle}{\partial x_{j}}+ \\
& v \frac{\partial^{2}\left\langle u_{j} T^{\prime} T^{\prime \prime}\right\rangle}{\partial x_{i} \partial x_{i}}-2 \in_{m i j} \Omega_{m}\left\langle u_{j} T^{\prime} T^{\prime \prime}\right\rangle \\
& \frac{\partial\left\langle T^{\prime} u_{j} T^{\prime \prime}\right\rangle}{\partial t^{\prime}}+\frac{\partial\left\langle u_{i}^{\prime} T^{\prime} u_{j} T^{\prime \prime}\right\rangle}{\partial x_{i}^{\prime}}=\left(\frac{v}{P_{r}}\right) \frac{\partial^{2}\left\langle T^{\prime} u_{j} T^{\prime \prime}\right\rangle}{\partial x_{i}^{\prime} \partial x_{i}^{\prime}}
\end{aligned}
$$

and

$$
\frac{\partial\left\langle T^{\prime \prime} u_{j} T^{\prime}\right\rangle}{\partial t^{\prime \prime}}+\frac{\partial\left\langle u_{i}^{\prime \prime} T^{\prime \prime} u_{j} T^{\prime}\right\rangle}{\partial x_{i}^{\prime \prime}}=\left(\frac{v}{P_{r}}\right) \frac{\partial^{2}\left\langle T^{\prime \prime} u_{j} T^{\prime}\right\rangle}{\partial x_{i}^{\prime \prime} \partial x_{i}^{\prime \prime}}
$$

Using the transformations

$$
\begin{aligned}
\frac{\partial}{\partial x_{i}}= & -\left(\frac{\partial}{\partial r_{i}}+\frac{\partial}{\partial r_{i}^{\prime}}\right), \frac{\partial}{\partial x_{i}^{\prime}}=\frac{\partial}{\partial r_{i}}, \frac{\partial}{\partial x_{i}^{\prime \prime}}=\frac{\partial}{\partial r_{i}^{\prime}} \\
\left(\frac{\partial}{\partial t}\right)_{t^{\prime}, t^{\prime \prime}} & =\left(\frac{\partial}{\partial t}\right)_{\Delta t, \Delta t^{\prime}}-\frac{\partial}{\partial \Delta t}-\frac{\partial}{\partial \Delta t^{\prime}}, \frac{\partial}{\partial \Delta t^{\prime}} \\
& =\frac{\partial}{\partial \Delta t}, \frac{\partial}{\partial \Delta t^{\prime \prime}}=\frac{\partial}{\partial \Delta t^{\prime}}
\end{aligned}
$$

into equations (17)-(19), we have

$$
\begin{gathered}
\frac{\partial\left\langle u_{j} T^{\prime} T^{\prime \prime}\right\rangle}{\partial t}-\left(\frac{\partial}{\partial r_{i}}+\frac{\partial}{\partial r_{i}^{\prime}}\right)\left\langle u_{j} T^{\prime} T^{\prime \prime} u_{i}\right\rangle+ \\
\frac{\partial\left\langle u_{i}^{\prime} T^{\prime} u_{j} T^{\prime \prime}\right\rangle}{\partial r_{i}}+\frac{\partial\left\langle u_{i}^{\prime \prime} T^{\prime \prime} u_{j} T^{\prime}\right\rangle}{\partial r_{i}^{\prime}} \\
=\frac{1}{\rho}\left(\frac{\partial}{\partial r_{j}}+\frac{\partial}{\partial r_{j}^{\prime}}\right)\left\langle P T^{\prime} T^{\prime \prime}\right\rangle+v\left(\frac{\partial}{\partial r_{i}}+\frac{\partial}{\partial r_{i}^{\prime}}\right)^{2}\left\langle u_{j} T^{\prime} T^{\prime \prime}\right\rangle+ \\
\left(\frac{v}{P_{r}}\right)\left(\frac{\partial^{2}}{\partial r_{i} \partial r_{i}}+\frac{\partial^{2}}{\partial r_{i}^{\prime} \partial r_{i}^{\prime}}\right)\left\langle u_{j} T^{\prime} T^{\prime \prime}\right\rangle-2 \epsilon_{m i j} \Omega_{m}\left\langle u_{j} T^{\prime} T^{\prime \prime}\right\rangle-
\end{gathered}
$$

$$
\frac{\partial\left\langle u_{j} T^{\prime} T^{\prime \prime}\right\rangle}{\partial \Delta t}+\frac{\partial\left\langle u_{i}^{\prime} T^{\prime} u_{j} T^{\prime \prime}\right\rangle}{\partial r_{i}}=\left(\frac{v}{P_{r}}\right) \frac{\partial^{2}\left\langle T^{\prime} u_{j} T^{\prime \prime}\right\rangle}{\partial r_{i} \partial r_{i}}
$$


$\frac{\partial\left\langle T^{\prime} u_{j} T^{\prime \prime}\right\rangle}{\partial \Delta t^{\prime}}+\frac{\partial\left\langle u_{i}^{\prime \prime} T^{\prime \prime} u_{j} T^{\prime}\right\rangle}{\partial r_{i}^{\prime}}=\left(\frac{v}{P_{r}}\right) \frac{\partial^{2}\left\langle u_{j} T^{\prime \prime} T^{\prime \prime}\right\rangle}{\partial r_{i}^{\prime} \partial r_{i}^{\prime}}$

Using the six-dimensional Fourier transforms of the type

$$
\begin{gathered}
\left\langle u_{j} T^{\prime} T^{\prime \prime}\right\rangle\left(\hat{r}, \hat{r}^{\prime}, \Delta t, \Delta t^{\prime}, t\right)=\int_{-\infty}^{\infty} \int_{-\infty}^{\infty}\left\langle\beta_{j} \theta^{\prime} \theta^{\prime \prime}\right\rangle \\
\exp \left[\hat{i}\left(\hat{K} \cdot \hat{r}+\hat{K}^{\prime} . \hat{r}^{\prime}\right)\right] d \hat{K} d \hat{K}^{\prime} \\
\left\langle u_{i} u_{j} T^{\prime} T^{\prime \prime}\right\rangle\left(\hat{r}, \hat{r}^{\prime}, \Delta t, \Delta t^{\prime}, t\right)=\int_{-\infty-\infty}^{\infty} \int_{-\infty}^{\infty}\left\langle\beta_{i} \beta_{j} \theta^{\prime} \theta^{\prime \prime}\right\rangle \\
\exp \left[\hat{i}\left(\hat{K} \cdot \hat{r}+\hat{K}^{\prime} . \hat{r}^{\prime}\right)\right] d \hat{K} d \hat{K}^{\prime}--(24) \\
\left\langle p T^{\prime} T^{\prime \prime}\right\rangle\left(\hat{r}, \hat{r}^{\prime}, \Delta t, \Delta t^{\prime}, t\right)=\int_{-\infty}^{\infty}\left\langle\alpha \theta^{\prime} \theta^{\prime \prime}\right\rangle \\
\exp \left[\hat{i}\left(\hat{K} . \hat{r}+\hat{K}^{\prime} \cdot \hat{r}^{\prime}\right)\right] d \hat{K} d \hat{K}^{\prime}---(25)
\end{gathered}
$$

Interchanging the points $p^{\prime}$ and $p^{\prime \prime}$ shows that

$$
\begin{array}{r}
\left\langle u_{j} u_{i}^{\prime \prime} T^{\prime} T^{\prime \prime}\right\rangle=\left\langle u_{j} u_{i}^{\prime} T^{\prime} T^{\prime \prime}\right\rangle=\int_{-\infty-\infty}^{\infty} \int_{j}^{\infty}\left\langle\beta_{j} \beta_{i}^{\prime} \theta^{\prime} \theta^{\prime \prime}\right\rangle \\
\exp \left[\hat{i}\left(\hat{K} . \hat{r}+\hat{K}^{\prime} . \hat{r}^{\prime}\right)\right] d \hat{K} d \hat{K}^{\prime}
\end{array}
$$

By use of these facts we can write equations (20)-(22) in the form

$$
\begin{gathered}
\frac{\partial\left\langle\beta_{j} \theta^{\prime} \theta^{\prime \prime}\right\rangle}{\partial t}\left(\hat{K}, \hat{K}^{\prime}, \Delta t, \Delta t^{\prime}, t\right)+\frac{v}{p_{r}}\left[\left(1+p_{r}\right) k^{2}+2 p_{r} k_{i} k_{i}^{\prime}+\right. \\
\left.\left(1+p_{r}\right) k^{\prime 2}+\frac{2 p_{r}}{v} \in_{m i j} \Omega_{m}\right]\left\langle\beta_{j} \theta^{\prime} \theta^{\prime \prime}\right\rangle \\
\left(\hat{K}, \hat{K}^{\prime}, \Delta t, \Delta t^{\prime}, t\right)=\frac{1}{\rho} i\left(k_{j}+k_{j}^{\prime}\right)\left\langle\alpha \theta^{\prime} \theta^{\prime \prime}\right\rangle+i\left(k_{i}+k_{i}^{\prime}\right) \\
{\left[\left\langle\beta_{i} \beta_{j} \theta^{\prime} \theta^{\prime \prime}\right\rangle-\left\langle\beta_{i}^{\prime} \beta_{j} \theta^{\prime} \theta^{\prime \prime}\right\rangle\left(\hat{K}, \hat{K}^{\prime}, \Delta t, \Delta t^{\prime}, t\right)--(27)\right.}
\end{gathered}
$$

$\frac{\partial\left\langle\beta_{j} \theta^{\prime} \theta^{\prime \prime}\right\rangle\left(\hat{K}, \hat{K}^{\prime}, \Delta t, \Delta t^{\prime}, t\right)}{\partial \Delta t}+\left(\frac{v}{p_{r}}\right) k^{2}\left\langle\beta_{j} \theta^{\prime} \theta^{\prime \prime}\right\rangle$

$\left(\hat{K}, \hat{K}^{\prime}, \Delta t, \Delta t^{\prime}, t\right)=-i k_{i}\left\langle\beta_{i}^{\prime} \beta_{j} \theta^{\prime} \theta^{\prime \prime}\right\rangle\left(\hat{K}, \hat{K}^{\prime}, \Delta t, \Delta t^{\prime}, t\right)--(28)$ $\frac{\partial\left\langle\beta_{j} \theta^{\prime} \theta^{\prime \prime}\right\rangle\left(\hat{K}, \hat{K}^{\prime}, \Delta t, \Delta t^{\prime}, t\right)}{\partial \Delta t^{\prime}}+\left(\frac{v}{p_{r}}\right) k^{\prime 2}\left\langle\beta_{j} \theta^{\prime} \theta^{\prime \prime}\right\rangle$

$\left(\hat{K}, \hat{K}^{\prime}, \Delta t, \Delta t^{\prime}, t\right)=-i k_{i}^{\prime}\left\langle\beta_{i}^{\prime} \beta_{j} \theta^{\prime} \theta^{\prime \prime}\right\rangle\left(\hat{K}, \hat{K}^{\prime}, \Delta t, \Delta t^{\prime}, t\right)--(29)$

If the derivative with respect to $x_{j}$ is taken of the momentum equation (14) for the point $p$, the equation multiplied by $T^{\prime} T^{\prime \prime}$ and taken the ensemble average, the resulting equation is

$$
\frac{\partial^{2}\left\langle u_{i} u_{j} T^{\prime} T^{\prime \prime}\right\rangle}{\partial x_{j} \partial x_{i}}=-\frac{1}{\rho} \frac{\partial^{2}\left\langle p T^{\prime} T^{\prime \prime}\right\rangle}{\partial x_{j} \partial x_{j}}
$$

Writing this equation in terms of the independent variables $\hat{r}$ and $\hat{r}^{\prime}$

$$
\begin{gathered}
{\left[\frac{\partial^{2}}{\partial r_{i} \partial r_{i}}+\frac{\partial^{2}}{\partial r_{i} \partial r_{i}^{\prime}}+\frac{\partial^{2}}{\partial r_{i}^{\prime} \partial r_{j}}+\frac{\partial^{2}}{\partial r_{i}^{\prime} \partial r_{j}^{\prime}}\right]\left\langle u_{i} u_{j} T^{\prime} T^{\prime \prime}\right\rangle=} \\
-\frac{1}{\rho}\left[\frac{\partial^{2}}{\partial r_{j} \partial r_{j}}+2 \frac{\partial^{2}}{\partial r_{j} \partial r_{j}^{\prime}}+\frac{\partial^{2}}{\partial r_{j}^{\prime}}\right]\left\langle p T^{\prime} T^{\prime \prime}\right\rangle-
\end{gathered}
$$

Taking the Fourier transforms of equation (31)

$$
\begin{aligned}
& \left\langle\alpha \theta^{\prime} \theta^{\prime \prime}\right\rangle= \\
& \frac{-\rho\left(k_{i} k_{j}+k_{i} k_{j}^{\prime}+k_{i}^{\prime} k_{j}+k_{i}^{\prime} k_{j}^{\prime}\right)\left\langle\beta_{i} \beta_{j} \theta^{\prime} \theta^{\prime \prime}\right\rangle}{k_{j} k_{j}+2 k_{j} k_{j}^{\prime}+k_{j}^{\prime} k_{j}^{\prime}}
\end{aligned}
$$

Equation (32) can be used to eliminate $\left\langle\alpha \theta^{\prime} \theta^{\prime \prime}\right\rangle$ from equation (27).

\section{Solution for times before the final period}

To obtain the equation for times before the final period of decay, the three point correlations are considered and the 
quadruple correlation terms decays faster than the lower-order correlation terms. If this assumption is made the equation (32) shows that the term $\left\langle\alpha \theta^{\prime} \theta^{\prime \prime}\right\rangle$ associated with the pressure fluctuations should also be neglected. Thus neglecting all the terms on the right hand side of equations (27)-(29).

$$
\begin{gathered}
\frac{\left.\partial\left\langle\beta_{j} \theta \theta^{\prime}\right\rangle \hat{K}, \hat{K}^{\prime}, \Delta t, \Delta \Delta^{\prime}, t\right)}{\partial t}+\frac{v}{p_{r}}\left[\left(1+p_{r}\right) k^{2}+2 p_{r} k_{i} k_{i}^{\prime}+\frac{2 p_{r}}{v} \epsilon_{m i j} \Omega_{m}\right] \\
\left\langle\beta_{j} \theta \theta^{\prime}\right\rangle\left(\hat{K}, \hat{K}^{\prime}, \Delta t, \Delta^{\prime}, t\right)=0 \\
\frac{\partial\left\langle\beta_{j} \theta^{\prime} \theta^{\prime \prime}\right\rangle\left(\hat{K}, \hat{K}^{\prime}, \Delta t, \Delta t^{\prime}, t\right)}{\partial \Delta t}+\left(\frac{v}{p_{r}}\right) k^{2}\left\langle\beta_{j} \theta^{\prime} \theta^{\prime \prime}\right\rangle \\
\left(\hat{K}, \hat{K}^{\prime}, \Delta t, \Delta t^{\prime}, t\right)=0 \\
\frac{\partial\left\langle\beta_{j} \theta^{\prime} \theta^{\prime \prime}\right\rangle\left(\hat{K}, \hat{K}^{\prime}, \Delta t, \Delta t^{\prime}, t\right)}{\partial \Delta t^{\prime}}+\left(\frac{v}{p_{r}}\right) k^{\prime 2}\left\langle\beta_{j} \theta^{\prime} \theta^{\prime \prime}\right\rangle \\
\left(\hat{K}, \hat{K}^{\prime}, \Delta t, \Delta t^{\prime}, t\right)=0
\end{gathered}
$$

Inner multiplication of equations (33), (34) and (35) by $\mathrm{k}_{\mathrm{j}}$ and integrating between $\mathrm{t}_{\mathrm{o}}$ and $t$ we obtain

$$
\begin{aligned}
& k_{j}\left\langle\beta_{j} \theta \theta^{\prime \prime}\right\rangle=f_{j} \exp \left\{-\frac{v}{p_{r}}\right. \\
& \left.\left[\left(1+\mathrm{p}_{\mathrm{r}}\right)\left(\mathrm{k}^{2}+\mathrm{k}^{2}\right)+2 \mathrm{p}_{\mathrm{r}} \mathrm{kk}^{\prime} \cos \theta+\frac{2 \mathrm{p}_{\mathrm{r}}}{v} \in_{\mathrm{mij}} \Omega_{\mathrm{m}}\right]\left(\mathrm{t}-\mathrm{t}_{0}\right)\right\}
\end{aligned}
$$

$k_{j}\left\langle\beta_{j} \theta^{\prime} \theta^{\prime \prime}\right\rangle=g_{j} \exp \left(-\frac{v}{p_{r}} k^{2} \Delta t\right)$

and

$$
k_{j}\left\langle\beta_{j} \theta^{\prime} \theta^{\prime \prime}\right\rangle=q_{j} \exp \left(-\frac{v}{p_{r}} k^{\prime 2} \Delta t^{\prime}\right)
$$

For these relations to be consistent, we have

$$
\begin{aligned}
& k_{j}\left\langle\beta_{j} \theta^{\prime} \theta^{\prime \prime}\right\rangle=k_{j}\left\langle\beta_{j} \theta^{\prime} \theta^{\prime \prime}\right\rangle_{0} \\
& \exp \left[-\frac{v}{p_{r}}\left\{\left(1+p_{r}\right)\left(k^{2}+k^{\prime 2}\right)\left(t-t_{0}\right)+k^{2} \Delta t+k^{\prime 2} \Delta t^{\prime}+\right.\right. \\
& \left.\left.2 p_{r} k k^{\prime} \cos \theta\left(t-t_{0}\right)+\frac{2 p_{r}}{v} \in_{m i j} \Omega_{m}\left(t-t_{0}\right)\right)\right]----(39)
\end{aligned}
$$

where $\theta$ is the angle between $\mathrm{k}$ and $k^{\prime}$ and $\left\langle\beta_{j} \theta^{\prime} \theta^{\prime \prime}\right\rangle_{0}$ is the value of $\left\langle\beta_{j} \theta^{\prime} \theta^{\prime \prime}\right\rangle$ at $\mathrm{t}=\mathrm{t}_{0}$, $\Delta t=\Delta t^{\prime}=0$. Letting $\hat{r}^{\prime}=0, \Delta t^{\prime}=0$ in the equation (23) and comparing the result with the equation (10) shows that

$$
\begin{aligned}
& \left\langle k_{j} \phi_{j} \tau \tau^{\prime}(\hat{K}, \Delta t, t)\right\rangle= \\
& \int_{-\infty}^{\infty}\left\langle k_{j} \beta_{j} \theta^{\prime} \theta^{\prime \prime}\left(\hat{K}, \hat{K}^{\prime}, \Delta t, 0, t\right) d \hat{K}\right. \text { - - - }
\end{aligned}
$$

Substituting the equation (39) and (40) into the equation (12) we obtain

$\frac{\partial\left\langle\tau \tau^{\prime}\right\rangle(\hat{K}, \Delta t, t)}{\partial t}+2 \frac{v}{p_{r}} k^{2}\left\langle\tau \tau^{\prime}\right\rangle(\hat{K}, \Delta t, t)=$

$\int_{-\infty}^{\infty} i k_{j}\left[\left\langle\beta_{j} \theta^{\prime} \theta^{\prime \prime}\right\rangle\left(\hat{K}, K^{\prime}, \Delta t, 0, t\right)-\left\langle\beta_{j} \theta^{\prime} \theta^{\prime \prime}\right\rangle\left(-\hat{K},-\hat{K}^{\prime}, \Delta t, 0, t\right)\right]_{0}$

$\exp \left[-\frac{v}{p_{r}}\left\{\left(1+p_{r}\right)\left(k^{2}+K^{\prime 2}\right)\left(t-t_{0}\right)+k^{2} \Delta t+k^{\prime 2} \Delta t^{\prime}+\right.\right.$

$\left.\left.2 p_{r} k k^{\prime}\left(t-t_{0}\right) \cos \theta+\frac{2 p_{r}}{v} \in_{m i j} \Omega_{m}\left(t-t_{0}\right)\right\}\right] d \hat{K}^{\prime}-$

Now, $d \hat{K}^{\prime}$ can be expressed in terms of $k^{\prime}$ and $\theta$ as $-2 \pi k^{\prime 2} d(\cos \theta) d \hat{k}^{\prime} \quad$ (cf.

Deissler $\left.{ }^{2}\right)$.

$$
\begin{aligned}
& \frac{\partial\left\langle\tau \tau^{\prime}\right\rangle(\hat{K}, \Delta t, t)}{\partial t}+2 \frac{v}{p_{r}} k^{2}\left\langle\tau \tau^{\prime}\right\rangle(\hat{K}, \Delta t, t) \\
& =2 \int_{-\infty}^{\infty} 2 \pi i k_{j}\left[\left\langle\beta_{j} \theta^{\prime} \theta^{\prime \prime}\right\rangle\left(k, k^{\prime}\right)-\left\langle\beta_{j} \theta^{\prime} \theta^{\prime \prime}\right\rangle\left(-\hat{K}, \hat{K}^{\prime}\right)\right]_{0}
\end{aligned}
$$




$$
\begin{gathered}
k^{\prime 2}\left[\int _ { - 1 } ^ { 1 } \operatorname { e x p } \left\{-\frac{v}{p_{r}}\left[\left(1+p_{r}\right)\left(k^{2}+k^{\prime 2}\right)\left(t-t_{0}\right)\right.\right.\right. \\
\quad+k^{2} \Delta t+k^{\prime 2} \Delta t^{\prime}+2 p_{r} k k^{\prime}\left(t-t_{0}\right) \cos \theta+ \\
\left.\left.\left.\frac{2 p_{r}}{v} \in_{m i j} \Omega_{m}\left(t-t_{0}\right)\right]\right\} d(\cos \theta)\right] d \hat{k}^{\prime}--(42)
\end{gathered}
$$

The quantity $\left[\left\langle\beta_{j} \theta^{\prime} \theta^{\prime \prime}\right\rangle\left(\hat{K}, k^{\prime}\right)-\left\langle\beta_{i}^{\prime} \theta^{\prime} \theta^{\prime \prime}\right\rangle\left(-\hat{K},-\hat{K}^{\prime}\right)\right]_{0}$ depends on the initial conditions of the turbulence.

In order to find the solution completely and following Loeffler and Deissler ${ }^{3}$ we assume that

$$
\begin{aligned}
i k_{j} & \left|\left\langle\beta_{j} \theta^{\prime} \theta^{\prime \prime}\right\rangle\left(\hat{K}, \hat{K}^{\prime}\right)-\left\langle\beta_{j} \theta^{\prime} \theta^{\prime \prime}\right\rangle\left(-\hat{K},-\hat{K}^{\prime}\right)\right|_{0} \\
& =-\frac{\delta_{0}}{(2 \pi)^{2}}\left(k^{2} k^{\prime 4}-k^{4} k^{\prime 2}\right)
\end{aligned}
$$

where $\delta_{0}$ is a constant depending on the initial condition. The negative sign is placed in front of $\delta_{0}$ in order to make the transfer of energy from small to large wave numbers for positive value of $\delta_{0}$.

Substituting equation (43) into equation (42), we get

$$
\begin{aligned}
& \frac{\partial}{\partial t}\left\langle\tau \tau^{\prime}\right\rangle(\hat{K}, \Delta t, t) \cdot 2 \pi+2 \frac{v}{p_{r}} 2 \pi k^{2}\left\langle\tau \tau^{\prime}\right\rangle(\hat{K}, \Delta t, t) \\
& =-2 \delta_{o} \int_{0}^{\infty}\left(k^{2} k^{\prime 4}-k^{4} k^{\prime 2}\right) k^{\prime 2} \\
& \times\left[\int _ { - 1 } ^ { 1 } \operatorname { e x p } \left\{-\frac{v}{p_{r}}\left[\left(1+p_{r}\right)\left(k^{2}+k^{\prime 2}\right)\left(t-t_{o}\right)+\right.\right.\right. \\
& k^{2} \Delta t+k^{\prime 2} \Delta t^{\prime}+2 p_{r} k k^{\prime}\left(t-t_{o}\right) \cos \theta
\end{aligned}
$$

$$
\left.\left.\left.+\frac{2 p_{r}}{v} \in_{m i j} \Omega_{m}\left(t-t_{o}\right)\right]\right\} d(\cos \theta)\right] d \hat{k}^{\prime}-(44
$$

Multiplying both side of equation (44) by $\mathrm{k}^{2}$, we get

$$
\frac{\partial E}{\partial t}+2 \frac{v}{p_{r}} k^{2} E=w
$$

where $E=2 \pi k^{2}\left\langle\tau \tau^{\prime}\right\rangle$, the energy spectrum function and $\mathrm{w}$ is given by

$$
\begin{aligned}
& w=-2 \delta_{o} \int_{0}^{\infty}\left(k^{2} k^{\prime 4}-k^{4} k^{\prime 2}\right) k^{2} k^{\prime 2} \\
& {\left[\int _ { - 1 } ^ { 1 } \operatorname { e x p } \left\{-\frac{v}{p_{r}}\left[\left(1+p_{r}\right)\left(k^{2}+k^{\prime 2}\right)\left(t-t_{o}\right)+k^{2} \Delta t\right.\right.\right.} \\
& \left.\left.+2 p_{r} k k^{\prime}\left(t-t_{o}\right) \cos \theta+\frac{2 p_{r}}{v} \in_{m i j} \Omega_{m}\left(t-t_{o}\right)\right]\right\} \\
& d(\cos \theta)] d \hat{k}^{\prime}
\end{aligned}
$$

Integrating equation (44) with respect to $\theta$, we have

$$
w=-\frac{\delta_{o}}{v\left(t-t_{o}\right)} \int_{0}^{\infty}\left(k^{3} k^{\prime 5}-k^{5} k^{\prime 3}\right)
$$

$$
\left[\operatorname { e x p } \left\{-\frac{v}{p_{r}}\left[\left(1+p_{r}\right)\left(k^{2}+k^{\prime 2}\right)\left(t-t_{o}\right)+k^{2} \Delta t\right.\right.\right.
$$$$
\left.\left.\left.-2 p_{r} k k^{\prime}\left(t-t_{o}\right)+\frac{2 p_{r}}{v} \in_{m i j} \Omega_{m}\left(t-t_{o}\right)\right]\right\}\right]
$$$$
d k^{\prime}+\frac{\delta_{o}}{v\left(t-t_{o}\right)} \int_{0}^{\infty}\left(k^{3} k^{\prime 5}-k^{5} k^{\prime 3}\right)
$$$$
\left[\operatorname { e x p } \left\{-\frac{v}{p_{r}}\left[\left(1+p_{r}\right)\left(k^{2}+k^{\prime 2}\right)\left(t-t_{o}\right)+k^{2} \Delta t+\right.\right.\right.
$$

$\left.\left.\left.2 p_{r} k k^{\prime}\left(t-t_{o}\right)+\frac{2 p_{r}}{v} \in_{m i j} \Omega_{m}\left(t-t_{o}\right)\right]\right\}\right] d k^{\prime} \quad--(47)$ 
Again integrating equation (47) with respect to $k^{\prime}$, we have

$$
\begin{aligned}
& w=-\frac{\delta_{o} \sqrt{\pi} p_{r}^{5 / 2}}{4 v^{3 / 2}\left(t-t_{o}\right)^{3 / 2}\left(1+p_{r}\right)^{5 / 2}} \exp \left\{-\frac{2 p_{r}}{v} \in_{m i j} \Omega_{m}\right. \\
& \left.\left(t-t_{o}\right)\right\} \times \exp \left[\frac{-k^{2} v\left(1+2 p_{r}\right)}{p_{r}\left(1+p_{r}\right)}\left(t-t_{o}+\frac{1+p_{r}}{1+2 p_{r}} \Delta t\right]\right. \\
& \times\left[\frac{15 p_{r} k^{4}}{4 v^{2}\left(t-t_{o}\right)^{2}\left(1+p_{r}\right)}+\left\{\frac{5 p_{r}^{2}}{\left(1+p_{r}\right)^{2}}-\frac{3}{2}\right\} \frac{k^{6}}{v\left(t-t_{o}\right)}\right. \\
& \left.+\left\{\frac{p_{r}^{3}}{\left(1+p_{r}\right)^{3}}-\frac{p_{r}}{1+p_{r}}\right\} k^{8}\right] \\
& -\frac{\delta_{o} \sqrt{\pi} p_{r}^{5 / 2}}{4 v^{3 / 2}\left(t-t_{o}+\Delta t\right)^{3 / 2}\left(1+p_{r}\right)^{5 / 2}} \exp \left\{-\frac{2 p_{r}}{v} \in_{m i j} \Omega_{m}\right. \\
& \left.\left(t-t_{o}\right)\right\} \times \exp \left[\frac{-k^{2} v\left(1+2 p_{r}\right)}{p_{r}\left(1+p_{r}\right)}\left(t-t_{o}+\frac{p_{r}}{1+2 p_{r}} \Delta t\right]\right. \\
& \times\left[\frac{15 p_{r} k^{4}}{4 v^{2}\left(t-t_{o}+\Delta t\right)^{2}\left(1+p_{r}\right)}+\left\{\frac{5 p_{r}^{2}}{\left(1+p_{r}\right)^{2}}-\frac{3}{2}\right\}\right. \\
& \left.\frac{k^{6}}{v\left(t-t_{o}+\Delta t\right)}+\left\{\frac{p_{r}^{3}}{\left(1+p_{r}\right)^{3}}-\frac{p_{r}}{1+p_{r}}\right\} k^{8}\right]--(48)
\end{aligned}
$$

The series of equation (46) contains only even power of $\mathrm{k}$ and start with $\mathrm{k}^{4}$.

If we integrate equation (48) for $\Delta t=0$ over all wave numbers, we find that

$$
\int_{0}^{\infty} w d k=0
$$

which is indicating that the expression for $\mathrm{w}$ satisfies the condition of continuity and homogeneity. Physically it was to be expected as $\mathrm{w}$ is a measure of the energy transfer and the total energy transferred to all wave numbers must be zero.
The linear equation (45) can be solved to give

$$
\begin{aligned}
& \left.E=\exp \left[-\frac{2 v}{p_{r}} k^{2}\left(t-t_{o}+\frac{\Delta t}{2}\right)\right]\right] \operatorname{wexp}\left[2 \frac{v}{p_{r}} k^{2}\left(t-t_{o}+\frac{\Delta t}{2}\right)\right] d t+ \\
& J(k) \exp \left[-\frac{2 v}{p_{r}} k^{2}\left(t-t_{o}+\frac{\Delta t}{2}\right)\right]
\end{aligned}
$$

where $\quad J(k)=\frac{N_{o} k^{2}}{\pi}$ is a constant of integration.

Substituting the values of $\mathrm{w}$ from (48) and $\mathrm{J}(\mathrm{k})$ into the equation (50) and integrating with respect to $t_{0}$ we get

$$
\begin{aligned}
& E=\frac{N_{o} k^{2}}{\pi} \exp \left[-2 \frac{v}{p_{r}} k^{2}\left(t-t_{o}+\frac{\Delta t}{2}\right)\right] \\
& +\frac{\delta_{o} \sqrt{\pi} p_{r}^{5 / 2}}{4 v^{3 / 2}\left(1+p_{r}\right)^{7 / 2}} \exp \left[-2 \epsilon_{m i j} \Omega_{m}\left(t-t_{o}\right)\right] \\
& \times \exp \left[\frac{-k^{2} v\left(1+2 p_{r}\right)}{p_{r}\left(1+p_{r}\right)}\left(t-t_{o}+\frac{1+p_{r}}{1+2 p_{r}} \Delta t\right)\right] \times \\
& {\left[\frac{3 p_{r} k^{4}}{2 v^{2}\left(t-t_{o}\right)^{5 / 2}}+\frac{p_{r}\left(7 p_{r}-6\right) k^{6}}{3 v\left(1+p_{r}\right)\left(t-t_{o}\right)^{3 / 2}}\right.}
\end{aligned}
$$

$$
\begin{aligned}
& -\frac{4\left(3 p_{r}^{2}-2 p_{r}+3\right) k^{8}}{3\left(1+p_{r}\right)^{2}\left(t-t_{o}\right)^{1 / 2}}+\frac{8 \sqrt{v}\left(3 p_{r}^{2}-2 p_{r}+3\right) k^{9}}{3\left(1+p_{r}\right)^{5 / 2} p_{r}^{1 / 2}} F(\eta) \\
& +\frac{\delta_{o} \sqrt{\pi} p_{r}^{5 / 2}}{4 v^{3 / 2}\left(1+p_{r}\right)^{7 / 2}} \times \exp \left[-2 \epsilon_{m i j} \Omega_{m}\left(t-t_{o}\right)\right] \\
& \times \exp \left[\frac{-v k^{2}\left(1+2 p_{r}\right)}{p_{r}\left(1+p_{r}\right)}\left(t-t_{o}+\frac{p_{r}}{1+2 p_{r}} \Delta t\right)\right. \\
& \times\left[\frac{3 p_{r} k^{4}}{2 v^{2}\left(t-t_{o}+\Delta t\right)^{5 / 2}}\right]+\frac{p_{r}\left(7 p_{r}-6\right) k^{6}}{3 v\left(1+p_{r}\right)\left(t-t_{o}+\Delta t\right)^{3 / 2}} \\
& -\frac{4}{3} \frac{\left(3 p^{2}{ }_{r}-2 p_{r}+3\right) k^{8}}{\left(1+p_{r}\right)^{2}\left(t-t_{o}+\Delta t\right)^{1 / 2}} \\
& +\frac{8 \sqrt{v}\left(3 p_{r}^{2}-2 p_{r}+3\right) k^{9}}{3\left(1+p_{r}\right)^{5 / 2} p_{r}^{1 / 2}} F(\eta)
\end{aligned}
$$


where $F(\eta)=\bar{e}^{\eta^{2}} \int_{0}^{\eta} e^{x^{2}} d x$

$$
\begin{array}{r}
\eta=k \sqrt{\frac{v\left(t-t_{o}\right)}{p_{r}\left(1+p_{r}\right)}} \\
\text { or } \quad \eta=k \sqrt{\frac{v\left(t-t_{o}+\Delta t\right)}{p_{r}\left(1+p_{r}\right)}}
\end{array}
$$

By setting $\hat{r}=0$ in equation (9) and use is made of the definition of $\mathrm{E}$, the result is

$$
\frac{\left\langle T T^{\prime}\right\rangle}{2}=\frac{\left\langle T^{2}\right\rangle}{2}=\int_{0}^{\infty} E d k
$$

Substituting equation (51) into equation (52) and integrating with respect to $\mathrm{k}$, gives

$$
\begin{array}{r}
\frac{\left\langle T^{2}\right\rangle}{2}=\frac{N_{o} p_{r}^{3 / 2}\left(T+\frac{\Delta T}{2}\right)^{-3 / 2}}{8 \sqrt{2 \pi} v^{3 / 2}}+ \\
\frac{\pi \delta_{o} p_{r}^{6}}{4 v^{6}\left(1+p_{r}\right)\left(1+2 p_{r}\right)^{5 / 2}} \exp \left[-2 \in_{m i j} \Omega_{m}\right] \\
\frac{\left[\frac{9}{16 T^{5 / 2}\left(T+\frac{1+p_{r}}{1+2 p_{r}} \Delta T\right)^{5 / 2}}+\right.}{16(T+\Delta T)^{5 / 2}\left(T+\frac{p_{r}}{1+2 p_{r}} \Delta T\right)^{5 / 2}}+ \\
\frac{5 p_{r}\left(7 p_{r}-6\right)}{16\left(1+2 p_{r}\right) T^{3 / 2}\left(T+\frac{1+p_{r}}{1+2 p_{r}} \Delta T\right)^{7 / 2}} \\
+\frac{5 p_{r}\left(7 p_{r}-6\right)}{16\left(1+2 p_{r}\right)(T+\Delta T)^{3 / 2}\left(T+\frac{p_{r}}{1+2 p_{r}} \Delta T\right)^{7 / 2}}
\end{array}
$$

$$
\begin{aligned}
& +\frac{35 p_{r}\left(3 p_{r}^{2}-2 p_{r}+3\right)}{8\left(1+2 p_{r}\right) T^{1 / 2}\left(T+\frac{1+p_{r}}{1+2 p_{r}} \Delta T\right)^{9 / 2}} \\
& +\frac{35 p_{r}\left(3 p_{r}^{2}-2 p_{r}+3\right)}{8\left(1+2 p_{r}\right)(T+\Delta T)^{1 / 2}\left(T+\frac{p_{r}}{1+2 p_{r}} \Delta T\right)^{9 / 2}} \\
& +\frac{8 p_{r}\left(3 p_{r}^{2}-2 p_{r}+3\right)\left(1+2 p_{r}\right)^{5 / 2}}{3.2^{23 / 2}\left(1+p_{r}\right)^{11 / 2}} \sum_{n=0}^{\infty} \frac{1.3 .5 \ldots \ldots \ldots .(2 n+9)}{n !(2 n+1) 2^{2 n}\left(1+p_{r}\right)^{n}}
\end{aligned}
$$$$
\left.\times\left\{\frac{T^{(2 n+1) / 2}}{(T+\Delta T / 2)^{(2 n+1) / 2}}+\frac{(T+\Delta T)^{(2 n+1) / 2}}{(T+\Delta T / 2)^{(2 n+1) / 2}}\right\}\right]
$$

where $\mathrm{T}=\mathrm{t}-\mathrm{t}_{0}$.

Equation (53) is the decay law of temperature energy fluctuations in homogeneous turbulence before the final period for the case of multi-point and multitime in a rotating system.

\section{Results and Discussion}

In equation (53) we obtained the decay law of temperature fluctuation in homogeneous turbulence before the final period in a rotating system neglecting quadruple correlation terms in comparison with the third -order terms for the case of multi-point and multi-time. If the system is non rotating then $\Omega_{\mathrm{m}}=0$ the equation (53) becomes

$$
\begin{aligned}
& \frac{\left\langle T^{2}\right\rangle}{2}=\frac{N_{o} p_{r}^{3 / 2}\left(T+\frac{\Delta T}{2}\right)^{-3 / 2}}{8 v^{3 / 2} \sqrt{2 \pi}}+\frac{\pi \delta_{o} p_{r}^{6}}{4 v^{6}\left(1+p_{r}\right)\left(1+2 p_{r}\right)^{5 / 2}} \\
& \times\left[\frac{9}{16 T^{5 / 2}\left(T+\frac{1+p_{r}}{1+2 p_{r}} \Delta T\right)^{5 / 2}}\right.
\end{aligned}
$$




$$
\begin{aligned}
& +\frac{9}{16(T+\Delta T)^{5 / 2}\left(T+\frac{p_{r}}{1+2 p_{r}} \Delta T\right)^{5 / 2}}+ \\
& \frac{5 p_{r}\left(7 p_{r}-6\right)}{16\left(1+2 p_{r}\right) T^{3 / 2}\left(T+\frac{1+p_{r}}{1+2 p_{r}} \Delta T\right)^{7 / 2}} \\
& +\frac{5 p_{r}\left(7 p_{r}-6\right)}{16\left(1+2 p_{r}\right)(T+\Delta T)^{3 / 2}\left(T+\frac{p_{r}}{1+2 p_{r}} \Delta T\right)^{7 / 2}} \\
& +\frac{35 p_{r}\left(3 p_{r}^{2}-2 p_{r}+3\right)}{8\left(1+2 p_{r}\right) T^{1 / 2}\left(T+\frac{1+p_{r}}{1+2 p_{r}} \Delta T\right)^{9 / 2}} \\
& +\frac{35 p_{r}\left(3 p_{r}^{2}-2 p_{r}+3\right)}{8\left(1+2 p_{r}\right)(T+\Delta T)^{1 / 2}\left(T+\frac{p_{r}}{1+2 p_{r}} \Delta T\right)^{9 / 2}} \\
& +\frac{8 p_{r}\left(3 p_{r}^{2}-2 p_{r}+3\right)\left(1+2 p_{r}\right)^{5 / 2}}{3.2^{23 / 2}\left(1+p_{r}\right)^{1 / 2}} \frac{1.3 .5 \ldots \ldots . .(2 n+9)}{n !(2 n+1)^{2 n}\left(1+p_{r}\right)^{n}} \\
& \left.\times\left\{\frac{T(2 n+1) / 2}{(T+\Delta T / 2)^{(2 n+1) / 2}}+\frac{(T+\Delta T)^{(2 n+1) / 2}}{(T+\Delta T / 2)^{(2 n+1) / 2}}\right\}\right]
\end{aligned}
$$

which is obtained earlier by Sarker and Islam. ${ }^{10}$

If we put $\Delta T=0$, we can easily find out

$$
\begin{aligned}
& \frac{\left\langle T^{2}\right\rangle}{2}=\frac{N_{o} p_{r}^{3 / 2} T^{-3 / 2}}{8 \sqrt{2 \pi} v^{3 / 2}}+\frac{\pi \delta_{o} p_{r}^{6} T^{-5}}{4 v^{6}\left(1+p_{r}\right)\left(1+2 p_{r}\right)^{5 / 2}} \times \\
& {\left[\frac{9}{16}+\frac{5}{16} \cdot \frac{p_{r}\left(7 p_{r}-6\right)}{1+2 p_{r}}+\frac{35}{8} \frac{p_{r}\left(3 p_{r}^{2}-2 p_{r}+3\right)}{\left(1+2 p_{r}\right)^{2}}+--\right]} \\
& =A T^{-3 / 2}+B T^{-5}=A\left(t-t_{0}\right)^{-3 / 2}+B\left(t-t_{0}\right)^{-5}-(55)
\end{aligned}
$$

where $A=\frac{N_{o} p_{r}^{3 / 2}}{8 \sqrt{2 \pi} v^{3 / 2}}$ and

$$
\begin{aligned}
& B=\frac{\pi \delta_{o} p_{r}^{6}}{2 v^{6}\left(1+p_{r}\right)\left(1+2 p_{r}\right)^{5 / 2}} \times\left[\frac{9}{16}+\frac{5}{16} \cdot \frac{p_{r}\left(7 p_{r}-6\right)}{1+2 p_{r}}\right. \\
& \left.+\frac{35}{8} \cdot \frac{p_{r}\left(3 p_{r}^{2}-2 p_{r}+3\right)}{\left(1+2 p_{r}\right)^{2}}+\ldots \ldots \ldots . .\right]
\end{aligned}
$$

which is obtained earlier by Loeffler and Deissler. $^{3}$

This study shows that the effect of rotation in homogeneous turbulence the temperature energy fluctuations decays more rapidly than the energy for non-rotating fluid for times before the final period.

If higher order correlation equation are considered in the analysis it appears that more terms of higher power of time would be added to the equation (53). For large times, the second term in the equation becomes negligible leaving the $-3 / 2$ power decay law for the final period.

\section{Acknowledgement}

The authors wishes to thank the Government of the People's Republic of Bangladesh for granting study leave and is also thankful to the Director General, Secondary and Higher Secondary Education, Bangladesh, Dhaka for his kind consideration. 


\section{References}

1. R. G. Deissler, On the decay of homogeneous turbulence before the final period, Phys. Fluid, 1 (1958) 111-121.

2. R. G. Deissler. A theory of decaying homogeneous turbulence, Phys. Fluid, 3 (1960) 176-187.

3. A. L. Loeffler and R. G. Deissler. Decay of temperature fluctuations in homogeneous turbulence before the final period. Int. J. Heat Mass Transfer, 1 (1961) 312 -324.

4. P. Kumar and S. R. Patel. One first order reactant in homogeneous turbulence before the final the period for the case of multipoint and single time, Phys. Fluid, 17 (1974) 1362.

5. P. Kumar and S. R. Patel. On first-order reactant in homogeneous turbulence. Int. J. Engeng. Sci., 13 (1975) 305-315.

6. S. R. Patel. On first-order reactants in homogeneous turbulence- numerical results. Int. J. Engeng. Sci., 14 (1976) 75-80.

7. M. S. A. Sarker and M. A. Islam. Decay of MHD turbulence before the final period for the case of multi-point and multi-time, Indian J. Pure appl. Math. 32(7) (2001) 1065-1076.

8. M. A. Islam and M. S. A. Sarker. First order reactant in MHD turbulence before the final period of decay for the case of multi-point and multi-time, Indian J. Pure appl. Math. 32(8) (2001) 1173-1184.

9. M. S. A. Sarker and M. L. Rahman. Decay of temperature fluctuations in MHD turbulence before the final period. North Bengal Univ. Review (Sci. and Technology), 9 (1998) 91.

10. M. S. A. Sarker and M. A. Islam. Decay of temperature fluctuations in homogeneous turbulence before the final period for the case of multi-point and multi-time, Ph. D. Thesis, Dept. of Mathematics, R.U., (2001) 27-40.

11. M. A. K. Azad and M. S. A. Sarker. Decay of MHD turbulence before the final period for the case of multi-point and multi-time in presence of dust particle. Bangladesh Journal of Scientific and Industrial Research 38(3-4) (2003) 151-164.

12. M. A. K. Azad and M. S. A. Sarker. Decay of dusty fluid MHD turbulence before the final period in a rotating system for the case of multi-point and multi-time. Journal of Bihar Mathematical Society, 23 (2003) $1-20$.

13. M. S. Sultana and M. S. A. Sarker. Decay of homogeneous dusty fluid turbulence before the final period, Jahangirnagar University Journal of Science, 27 (2004) 263-276.

14. M. A. K. Azad and M. S. A. Sarker. Statistical theory of certain distribution functions in MHD turbulent flow in a 
rotating system in presence of dust particles, Rajshahi University Studies, Part-B. Journal of science, 32 (2004) 193-210 (In Press).

15. M. S. A. Sarker and M. A. K. Azad. Decay of dusty fluid MHD turbulence before the final period for the case of multi-point and multi-time in a rotating system,
Rajshahi University Studies, Part-B, Journal of science, 32 (2004) 177-192 (In Press).

16. I. N. Sneddon. Fourier Transforms (McGraw -Hill Book Company, Inc., N. Y.), (1951) 43. 